\title{
Meat consumption: attitudes and beliefs of meat-eating, meat-reducing and non-meat-eating subjects in the Republic of Ireland
}

\author{
G. Doherty ${ }^{1}$, S. Cassidy ${ }^{1}$, I. Huybrechts ${ }^{2}$ and A. Mullee ${ }^{3}$ \\ ${ }^{1}$ UCD Institute of Food and Health, University College Dublin, Belfield, Dublin, Ireland, \\ ${ }^{2}$ Nutrition and Metabolism Section, International Agency for Research on Cancer, World Health Organization, Lyon, \\ France and \\ ${ }^{3}$ Department of Health and Nutrition Science, IT Sligo, Sligo, Ireland
}

A global shift towards plant-based diets has been proposed with a particular focus on a reduction in meat production and consumption. Environmental sustainability and human health are inextricably linked by diet ${ }^{(1)}$. It has been estimated that an individual's meat consumption should not exceed $98 \mathrm{~g} /$ week of red meat and $203 \mathrm{~g} /$ week of poultry in order to eat without causing any net damage to the environment ${ }^{(1)}$. Meat and meat products contribute to approximately seventeen-percent of Irish adults' daily energy intake, with red meat and poultry intake greater than these estimates ${ }^{(2)}$. The objective of this study was to investigate the attitudes and beliefs about meat consumption and vegetarianism amongst meat-eaters, meat-reducers, and non-meat-eaters in the Republic of Ireland to understand the motives behind individuals' dietary patterns.

An online questionnaire with multiple choice and open-ended questions was disseminated through an electronic newsletter and on social media. The questionnaire was open to adults aged $\geq 18$ years resident in the Republic of Ireland. Ethical Exemption was given by the University College Dublin Human Research Ethics Committee. Respondents were grouped into three categories meat-eaters (omnivores), meat-reducers (part-time vegetarians and nearly vegetarians) and non-meat-eaters (vegans, vegetarians and pescatarians). Chi square tests were used to assess the likelihood of reducing meat consumption in the future depending if the respondent had previously made any meat reduction actions.

A total of 325 individuals responded of which $78 \%$ were female and $26 \%$ reported not eating meat. Taste was the main reason for eating meat $(72 \%)$, followed by health reasons $(53 \%)$. While health was also stated as a main reason for following vegetarianism $(66 \%)$, the environment was the salient motive for following this dietary pattern $(71 \%)$, with $75 \%$ of all responders believing meat production was bad for the environment. This view varied according to dietary pattern where a higher proportion of non-meat-eaters $(94 \%)$ believed production was bad compared to meat-reducers $(84 \%)$ and meat-eaters $(60 \%)$. Approximately three-quarters $(77 \%)$ of respondents believed everyone should eat less red meat, while only $35 \%$ believed everyone should stop eating red and processed meat. Furthermore, $78 \%$ intend to reduce their meat consumption in the future. A significant association between whether the respondent had altered their meat consumption previously and their intention to reduce meat consumption in future was observed $(\mathrm{p}<0.01)$.

This study highlights motivators for following meat-eating or meat-reducing or non-meat-eating diets. As attitudes differ between dietary pattern groups, awareness of these varying motivators can help inform public health campaigns to reduce meat consumption in the Republic of Ireland and support a move towards more sustainable dietary patterns.

\section{References}

1. Willett W, Rockström J, Loken B, et al. (2019) The Lancet 393, 447-492

2. Cocking C, Kehoe L, McNulty BA, et al. (2018) Proc Nut Soc 77 (OCE3) 